\title{
Image Quality in Computed Tomography Coronary Angiography and Radiation Dose Reduction
}

\author{
Domas GOLUBICKAS ${ }^{1}$, Saulius LUKOSEVICIUS ${ }^{1}$, \\ Vytenis TAMAKAUSKAS ${ }^{2}$, Laima DOBROVOLSKIENE ${ }^{1}$, \\ Ingrida BASEVICIENE ${ }^{1}$, Liviu GRIB ${ }^{3}$, Nijole RAGAISYTE ${ }^{1}$, \\ Rytis LEONAVICIUS ${ }^{1}$, Viktor MEDVEDEV ${ }^{4}$, Vincentas VEIKUTIS ${ }^{2, *}$ \\ ${ }^{1}$ Lithuanian University of Health Sciences, Kaunas, Lithuania \\ 2 Institute of Cardiology, Lithuanian University of Health Sciences, Kaunas, Lithuania \\ ${ }^{3}$ State University of Medicine and Pharmacy "Nicolae Testemitanu”, Chisinau, Moldova \\ ${ }^{4}$ Institute of Data Science and Digital Technologies, Vilnius University, Vilnius, Lithuania \\ e-mail:domas.golubickas@lsmuni.lt,saulius.lukosevicius@kaunoklinikos.lt, \\ v.tamakauskas@gmail.com,laimadobra@gmail.com,ibasev@gmail.com,liviu.grib@usmf.md, \\ Nijole.Ragaisyte@lsmu.lt,rytis.leo@gmail.com,viktor.medvedev@mif.vu.lt, \\ vincentas.veikutis@lsmuni.lt
}

Received: July 2020; accepted: October 2021

\begin{abstract}
Computed tomography coronary angiography (CTCA) is a non-invasive, powerful image processing technique for assessing coronary artery disease. The aim of the paper is to evaluate the diagnostic role of CTCA using optimal scanning parameters and to investigate the effect of low kilovoltage CTCA on the qualitative and quantitative image parameters and radiation dose in overweight and obese patients. Consolidation of knowledge in medicine and image processing was used to achieve the aim, and performance was evaluated in a clinical setting. Elevated body mass index is one of the factors causing increased radiation dose to patients. This study examined the feasibility of $80-\mathrm{kV}$ and $100-\mathrm{kV}$ CTCA in overweight and obese adult patients, comparing radiation doses and image quality versus standardized $100-\mathrm{kV}$ protocols in the group of overweight patients and $120-\mathrm{kV}$ CTCA in the group of obese patients. Qualitative and quantitative image parameters were determined in proximal and distal segments of the coronary arteries. Quantitative assessment was determined by the contrast-to-noise ratio and signal-to-noise ratio. The results of the study showed that in overweight and obese patients, the low dose protocol affords radiation dose reduction of $35 \%$ and $41 \%$, respectively. Image quality was found to be diagnostically acceptable in all cases.
\end{abstract}

Key words: image quality, data analysis, computed tomography, coronary angiography, radiation dose, optimal scanning parameters.

\section{Introduction}

In recent years, there has been a rapid development of data analysis, computer science, and medicine. New research areas and new trends are emerging, such as data mining, knowl-

\footnotetext{
${ }^{*}$ Corresponding author.
} 
edge discovery, deep learning, and image processing. Data science is a multidisciplinary subject that includes data mining, big data, data analytics, machine learning, and data knowledge discovery. Data science combines three highly iterative research areas: mathematics/statistics/operational research, computer science, and digital technologies used to study and perceive data (Dzemyda, 2018). Data science is finding new applications: biotechnology, materials microscopy, geographic research, learning analytics, radiology, and many others. The multidisciplinary efforts of data scientists, medical doctors, regulators and health insurance organizations are increasingly required. Computational methods need to move forward to bring direct benefit to clinical practice.

Digital image processing provides automatic interpretation, manipulation, and analysis of visual information and includes image collection, processing, and analysis. Image processing technologies have many different applications, such as space image processing, medical or biological image processing, remote sensing and extracting details from microscopic images. Recently, an increasing number of diagnostic and treatment decisions in medicine are being made on the basis of image analysis (Budginaitè et al., 2021; Kielaite-Gulla et al., 2021). Images are obtained using clinical diagnostic techniques such as radiology, magnetic resonance, thermal imaging, tomography, etc. Image analysis is becoming an advanced technology to help make important diagnostic decisions in medicine (Pocè et al., 2021). Many diseases can be diagnosed and treated using computed tomography (CT), a technology that allows changes inside the human body to be observed without an incision using computer-processed X-rays (Bilinskas et al., 2017, 2018). A CT scan is a three-dimensional image, i.e. a set of two-dimensional images representing a crosssection of the human body in the transverse plane. Such a set of images requires specific techniques and tools for image data processing, for example, segmentation of images, medical modelling, image registration (Bilinskas et al., 2017). A CT scan should be performed before and after treatment to assess the efficacy of treatment or progress in the treatment of the disease.

Computed Tomography Coronary Angiography (CTCA) is a non-invasive worldwide approved imaging method and diagnostic tool for evaluating patients with suspected coronary artery disease (CAD). It's particularly useful for verification or exclusion of CAD because of established high sensitivity and negative predictive value (Budoff et al., 2008; Nasis et al., 2010). The basic principle of CTCA is to obtain volumetric data set through the heart during peak coronary artery enhancement. The final image quality and associated radiation exposure are determined by both technical and patient-dependent factors (Ghekiere et al., 2017). Data analysis often requires long time training and extreme proficiency. Up-to-date diagnosis of coronary artery disease is important for the prevention of cardiovascular disease through pharmacological treatment. However, coronary angiography is usually restricted to patients with a high probability of significant CAD, due to its relatively high costs and invasive nature with a small, but not negligible, risk of procedure-related, potentially life-threatening complications (Meijboom, 2009). Also, CT angiography-based diagnostic may provide clinically significant information to determinate stenosis of coronary arteries, stents or bypass grafts. In case of positive CTCA scan result (moderate or significant CAD), these patients should be referred for coronary angiography or surgical revascularization. 
When performing a CTCA procedure, it is important to select patients who require certain conditions. The factors which can be important for the data interpretations are associated with patient heart rate variability, image windowing parameters, and surely overweight, which can significantly lower specificity and sensitivity of the procedure and CT artifacts. Therefore, radiation exposure remains a serious limitation of radiological techniques based on X-rays, including CTCA, and it is very important to perform procedures in obese patients when higher radiation power and exposure than it is required for a natural procedure. Given the fact that X-ray diagnostic tests are used in many other fields of medical investigations, the overall size of the patient irradiance becomes sufficiently high. Many different methods of reducing radiation of different origins have been reported. The radiation dose required for CTCA in clinical practice has decreased significantly in recent years (Stocker et al., 2018). To minimize radiation exposure, recent advances include the advent use of wide detector scanners such as the 320-detector row scanner which has a $16-\mathrm{cm}$ cranial-caudal coverage and allows for acquisition of the whole coronary artery tree in one gantry rotation over less than one heartbeat (Nasis et al., 2010), therefore prospective triggered scanning protocol is applicable to achieve enough good diagnostic image quality with low but effective dose. Advances in scanner technology and better protocols permit lower radiation dose cardiac imaging with doses below $1 \mathrm{mSv}$ (Achenbach et al., 2011, 2009; Heilbron and Leipsic, 2010; Wang et al., 2012). In individuals with the suitable body size, a lower tube voltage setting in combination with iterative reconstruction algorithm may allow a reduction in radiation dose while maintaining the quality of the diagnostic image (Dalager et al., 2011; Feuchtner et al., 2010; Gagarina et al., 2011; Kalender et al., 2009; Oda et al., 2011; Wang et al., 2012), since the radiation exposure generally varies with the square of the tube potential (Huda et al., 2000). The use of $80-\mathrm{kV}$ has been proven feasible in adult patients with a body mass index (BMI) below $22.5 \mathrm{~kg} / \mathrm{m}^{2}$ (Oda et al., 2011; Wang et al., 2012). However, limited data is available to recommend size or weight thresholds for $80-\mathrm{kV}$ or $100-\mathrm{kV}$ in routine cardiac CT examinations. In addition, further research is needed to assess the accuracy of low $(80-\mathrm{kV})$ protocols to evaluate coronary artery disease, especially in small vessels, before they can be reliably implemented into widespread clinical practice for cardiac CT angiography (Engel et al., 2012). To date, we have not found any CTCA low dose studies, particularly involving overweight or really obese patients.

The aim of the paper is to evaluate the diagnostic role of CTCA-based solution using optimal scanning parameters and to investigate the effect of low kilovoltage CTCA on qualitative and quantitative image parameters and radiation dose in overweight and obese patients. The research was conducted to examine the feasibility of 80-kV CTCA in overweight adult patients group (BMI $25-30 \mathrm{~kg} / \mathrm{m}^{2}$ ) and $100-\mathrm{kV}$ CTCA in obese patients by comparing radiation doses, image quality and diagnostic opportunities versus common standardized 100-kV protocols in overweight and 120-kV CTCA in obese patient groups. 


\section{Materials and Methods}

\subsection{Scan Protocol}

Generally, all patients were noticed to avoid caffeine and smoking 12 hours, and eating solid food alike to increase fluid intake 4 hours before the exam procedure. The breathhold of 10 up to 20 seconds was manageable for most of the tested patients. Standard precautions with regard to contrast allergy history and renal function were routinely taken. On arrival, an 18-gauge intravenous line was inserted in the right antecubital vein for administration of contrast. Patients with heart rates of more than $70 \mathrm{bpm}$ received (unless with ECG atrioventricular conduction abnormalities) a single peroral dose of $50 \mathrm{mg}$ metoprolol $60 \mathrm{~min}$ before the scanning procedure. Sublingual nitroglycerine $(0.4 \mathrm{mg})$ was administered unless contraindicated. Patients with heart rates of more than $80 \mathrm{bpm}$ received an additional peroral dose of metoprolol (if not contraindicated). CTCA procedures were clinically indicated, performed as standard of care.

The scan was acquired during bolus injection of nonionic iodinated contrast material (Iopromide $370 \mathrm{mg} \mathrm{I} / \mathrm{ml}$ ) 66 at a flow rate of $6 \mathrm{~mL} / \mathrm{s}$ into the antecubital vein, followed by $40 \mathrm{~mL}$ of saline at $5 \mathrm{~mL} / \mathrm{s}$, during a single breathhold at slight inspiration. CTCA was performed on a 320-detector-row CT scanner (Aquilion ONE, Toshiba Medical Systems, Japan). The scanning parameters were as follows: detector collimation $320 \mathrm{~mm} \times 0.5 \mathrm{~mm}$; tube current $580 \mathrm{~mA}$; tube voltage 80 or $100-\mathrm{kV}$; gantry rotation time $350 \mathrm{~ms}$; and temporal resolution $175 \mathrm{~ms}$. Prospectively triggered scanning was used covering 70-80\% of the $\mathrm{R}-\mathrm{R}$ interval. The scan range covered the entire heart from the level of the carina to the diaphragm. Scanned images were reconstructed using AIDR 3D reconstruction, cardiac CTCA standard protocol (FC03 convolution filter).

\subsection{Data Analysis}

Radiation dose and data collection were carried out as follows: parameters including tube potential $(\mathrm{kV})$, tube current-time product (mAs), volume-weighted CT dose index (CTDIvol), dose-length product (DLP) and dose-related parameters, including heart rate, rhythm, use of $\beta$ blockers and nitroglycerine, the volume of contrast agent (cc) and flow rate $(\mathrm{cc} / \mathrm{sec})$, weight, height, BMI were recorded for each patient during the examination. Effective dose (ED, in units of $\mathrm{mSv}$ ) was calculated using the formulate $E D=D L P \times k$, where $k$ is the conversion factor $\left(k=0.014 \mathrm{mSv} \mathrm{mGy}^{-1} \mathrm{~cm}^{-1}\right)$ (Abbara et al., 2016). Measurement of the chest wall as a substitute for body-mass-index: a full field-of-view volume data of each patient was analysed at a 3D-imaging workstation (Vitrea, Toshiba, Japan) and manual measurement of a chest wall thickness was performed by a radiologist to measure the patient's chest wall thickness at the level of processus xiphoideus.

\subsection{Image Evaluation}

Qualitative assessment: CT data should be interpreted on a computer workstation capable of all post-processing methods (Vitrea, Toshiba, Japan). Qualitative image quality was determined retrospectively by use of a 5-point Likert scale for each coronary artery segment 
(5: very good image quality, no artifacts, sharply delineated contours of the vessel lumen; 4: fully diagnostic image quality, no artifacts, very minor blurring of the vessel lumen contours; 3: diagnostic image quality, moderate blurring of lumen contours; 2 : poor image quality; severe artifacts, prominent blurring of the contours; 1: non-diagnostic segment segment not visible due to artifacts). 1 and 2 points were considered as non-diagnostic. Segmentation of the coronary arteries was performed based on the American Heart Association 17-segment model. All 51 cases in the study were interpreted and overall qualitative image quality was determined. For assessment of the inter-observer agreement, four blinded readers with at least 3-10 years experience in cardiac CT imaging evaluated all coronary segments in 10 randomly selected patients. Prior to the subjective image quality readouts, the readers were informed on the criteria of image grading and assessed 5 test cases together.

Quantitative assessment: for quantitative image quality assessment, CT data sets were sent to the 3D-imaging workstation (Vitrea, Toshiba, Japan). To determine the signal-tonoise ratio (SNR) and contrast-to-noise ratio (CNR), the largest possible circular regions of interest [ROI] $\left(2-4 \mathrm{~mm}^{2}\right)$ were placed in the coronary lumen and the adjacent connective tissue of the vessel. The measurements were performed in nine different coronary segments: proximal and distal right coronary artery [AHA segment \#1 and \#3], left main [AHA segment \#5], proximal and distal left anterior descending [AHA segment \#6 and \#8], first diagonal branch [AHA segment \#9], proximal and distal left circumflex artery [AHA segment \#11 and \#14]. Within these regions of interest, the mean CT contrast attenuation was recorded. Image noise was defined as the standard deviation of CT density in a region of interest (ROI) placed in the aortic root at a position cranial to the left main coronary artery. CNR was obtained by dividing the difference in CT attenuation between the coronary lumen and surrounding tissue by the image noise. SNR was determined by dividing the contrast of the coronary lumen by the background noise as described previously (Feuchtner et al., 2010; Engel et al., 2012; Bittencourt et al., 2011; Pflederer et al., 2009; Wang et al., 2012; Leschka et al., 2008).

\subsection{Artifacts Correction}

In order to obtain phase-consistent images and simultaneously avoid motion artifacts, images were scanned and reconstructed during mid-end diastole (70-80 percent interval of the cardiac cycle) when cardiac motion is least. Fixed 75 percent phase and automatically by the vendor's dedicated algorithm selected phase with least motion artifacts were routinely evaluated. If the quality of coronary CT images is not satisfactory, additional manual reconstructions were made to find the best quality phase in the scanned interval. Short time irregular heart rhythm can create artifacts and result in deterioration of image quality. Motion artifacts most frequently occurred in the mid-right coronary artery (segment 2) position because this segment usually moves most rapidly. In the case of local segmental artifacts, reconstruction was performed in the best image phase of the segment using ECG-edit software of AIDR 3D.

In the presence of marked and outspread all coronary vessels' calcification, the correct stenosis interpretation is obscured in most cases. It is because scattered calcium in CT 
Table 1

Patient characteristics.

\begin{tabular}{lllll}
\hline Patient group & \multicolumn{2}{l}{ Group 1 (overweight) } & \multicolumn{2}{l}{ Group 2 (obese) } \\
\hline Tube kilovoltage (BMI) & $80 \mathrm{kV}$ & $100 \mathrm{kV}$ & $100 \mathrm{kV}$ & $120 \mathrm{kV}$ \\
& $\left(25-30 \mathrm{~kg} / \mathrm{m}^{2}\right)$ & $\left(25-30 \mathrm{~kg} / \mathrm{m}^{2}\right)$ & $\left(30-35 \mathrm{~kg} / \mathrm{m}^{2}\right)$ & $\left(30-35 \mathrm{~kg} / \mathrm{m}^{2}\right)$ \\
Age (years), mean $\pm \mathrm{SD}$, range & $59.4 \pm 12.4$ & $62 \pm 9.9$ & $61.2 \pm 12.5$ & $62.3 \pm 10.6$ \\
& $26-74$ & $49-86$ & $30-77$ & $39-83$ \\
Male gender, \% & $30.4(7 / 23)$ & $48.4(15 / 31)$ & $36.0(9 / 25)$ & $39.3(11 / 28)$ \\
Heart rate (bpm), mean $\pm \mathrm{SD}$ & $59.2 \pm 6.6$ & $59.5 \pm 4.9$ & $61.6 \pm 2.3$ & $58.6 \pm 5.2$ \\
Synus rhythm, \% & \multicolumn{2}{c}{$100(107 / 107)$} \\
BMI $\left(\mathrm{kg} / \mathrm{m}^{2}\right)$, mean $\pm \mathrm{SD}$, range & $26.8 \pm 1.2$ & $27.2 \pm 1.5$ & $32.2 \pm 1.5$ & $31.8 \pm 1.6$ \\
& $25.1-29.1$ & $25.0-29.7$ & $30.0-34.9$ & $30.1-34.9$ \\
Chest wall thikness $(\mathrm{min})$, mean $\pm \mathrm{SD}$ & $18.3 \pm 6.7$ & $19.0 \pm 8.1$ & $20.7 \pm 6.3$ & $24.2 \pm 6.6$ \\
\hline
\end{tabular}

images creates blooming and beam hardening artifacts and visualization of the underlying noncalcified plaque or often lumen is more complicated.

\subsection{Statistical Analysis}

Continuous variables were expressed as mean \pm standard deviations for normally distributed and median with interquartile range [IQR; 25th and 75th percentiles] for nonnormally distributed variables. Categorical variables were expressed as frequencies or percentages. The differences in continuous variables between the groups were compared using two-tailed Student's $t$-test (for normally distributed variables) or Wilcoxon test (for non-normally distributed variables). Categorical variables were compared using the chisquare test or Fisher's exact test. To determine the inter-observer agreement for the qualitative image quality assessment, intra-class-correlation (ICC) and Pearson's correlation coefficient were calculated. A $p$-value of less than 0.05 was considered statistically significant.

\section{Results}

\subsection{Baseline Data}

Table 1 presents the baseline patient characteristics. All patients were divided into two groups according to the body weight (the first group was overweight and the second obese), and each group was divided into two subgroups according to minimized or common tube kilovoltage $(80-\mathrm{kV}$ or $100-\mathrm{kV}$ in the first and $10-\mathrm{kV}$ or $120-\mathrm{kV}$ in the second). Body mass index limit $\left(\leqslant 35 \mathrm{~kg} / \mathrm{m}^{2}\right)$ was chosen due to chest wall thickness and artifacts, CT scanner gantry diameter and moving table technical options. There were no statistically significant differences inside the selected two groups for age, gender, body weight, BMI, heart rate and scan length. However, the groups differed according to the thickness of the chest wall, which is a key factor in determining the quality of the scan. All patients had sinus heart rhythm, and only in a few cases we observe several supraventricular or, rarely, ventricular extra-systoles what were successfully compromised by beta-blockers and did not affect 

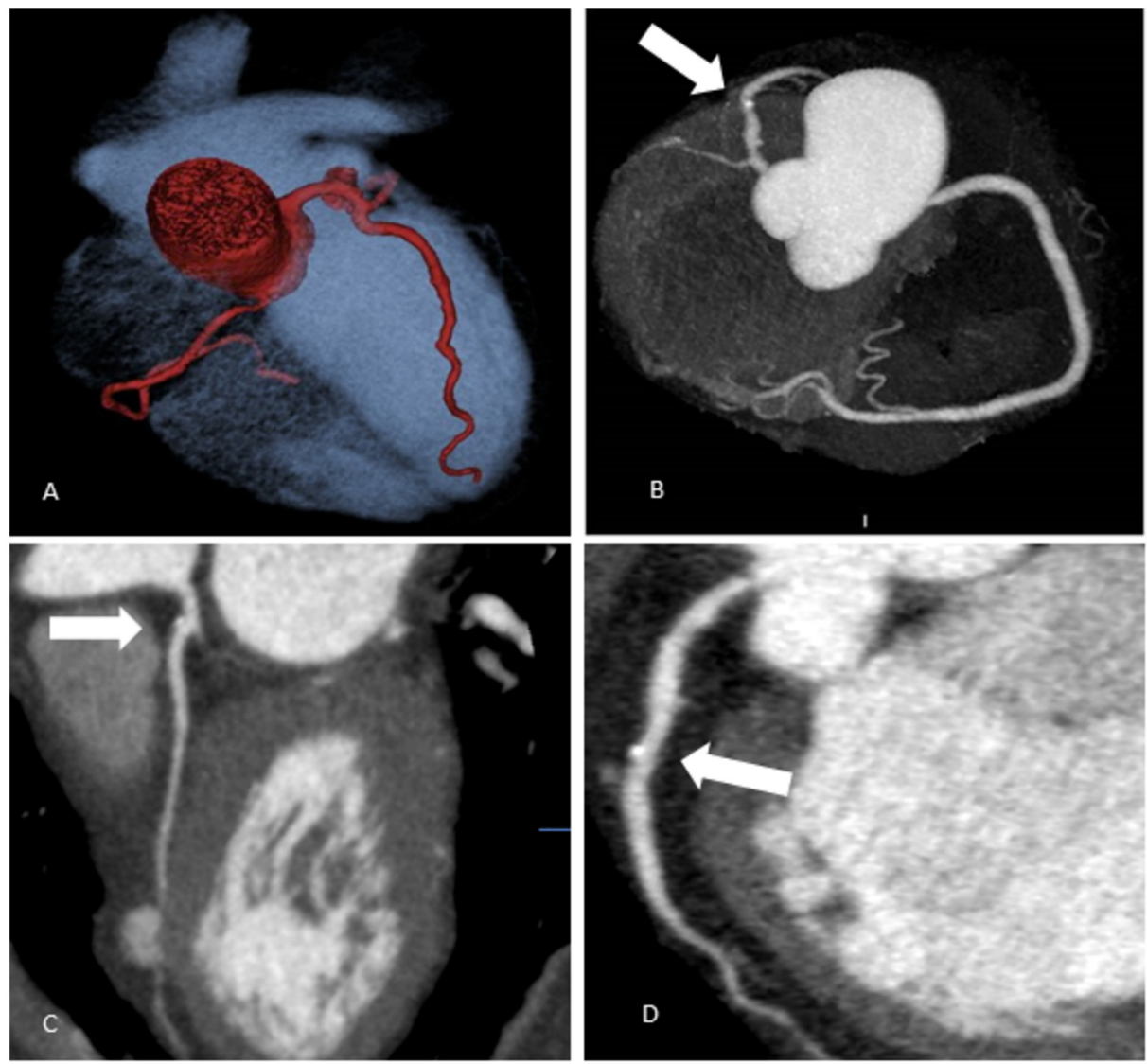

Fig. 1. Coronary arteries' scanning images (Group 1 - overweight patients).

A - Coronary arteries' 3D reconstruction. Patient's BMI $29.1 \mathrm{~kg} / \mathrm{m}^{2} ; 80-\mathrm{kV}$, effective dose $1.2 \mathrm{mSv}$.

B - Coronary arteries' 3D reconstruction. Patient's BMI $25.1 \mathrm{~kg} / \mathrm{m}^{2} ; 80-\mathrm{kV}$, effective dose $0.95 \mathrm{mSv}$; calcified plaque in LAD S6 (labelled by white arrow).

C - Multiplanar curved reconstruction of LAD coronary artery. LAD S6 mixed plaque, 20\% stenosis (labelled by white arrow). Patient's BMI $28.2 \mathrm{~kg} / \mathrm{m}^{2}, 100-\mathrm{kV}$.

D - Multiplanar curved reconstruction of LAD coronary artery. LAD S7 calcified plaque, 25\% stenosis (labelled by white arrow). Patient's BMI $25.1 \mathrm{~kg} / \mathrm{m}^{2}, 80-\mathrm{kV}$.

the results. Two patients have paroxysmal atrial fibrillation in anamnesis, but did not have it at the time of CTCA, i.e. they also had a stable sinus rhythm.

In our study, we tested the feasibility of low doses protocols $(80-\mathrm{kV}$ and $100-\mathrm{kV})$ depending on BMI, and demonstrated a significant reduction of radiation dose $35 \%$ and $41 \%$ versus standard $100-\mathrm{kV}$ and $120-\mathrm{kV}$ protocols: $(1.1 \mathrm{mSv}$ [0.9-1.2] $(80-\mathrm{kV})$ versus $1.7 \mathrm{mSv}$ [1.0-2.0] (100-kV) and $1.6 \mathrm{mSv}$ [1.4-1.9] (100-kV) versus $2.7 \mathrm{mSv}$ [2.1-3.0] $(120-\mathrm{kV}))$. Our data demonstrate that in an overweight patient group, an ultra-low dose protocol (radiation dose reduction of 35\%) scanning results are feasible enough. It allows the identification of patients with significant and intermediate CAD, as well as the identification of patients with no-, single-, or multi-vessel disease (see Fig. 1). The segmental 
Table 2

Results on qualitative image quality and radiation dose.

\begin{tabular}{|c|c|c|c|c|}
\hline \multirow{2}{*}{$\begin{array}{l}\text { Patient groups } \\
\text { Kilovoltage (BMI group) }\end{array}$} & \multicolumn{2}{|c|}{ Group 1 (overweight) } & \multicolumn{2}{|l|}{ Group 2 (obese) } \\
\hline & $\begin{array}{l}80-\mathrm{kV} \\
\left(25-30 \mathrm{~kg} / \mathrm{m}^{2}\right)\end{array}$ & $\begin{array}{l}100-\mathrm{kV} \\
\left(25-30 \mathrm{~kg} / \mathrm{m}^{2}\right)\end{array}$ & $\begin{array}{l}100-\mathrm{kV} \\
\left(30-35 \mathrm{~kg} / \mathrm{m}^{2}\right)\end{array}$ & $\begin{array}{l}120-\mathrm{kV} \\
\left(30-35 \mathrm{~kg} / \mathrm{m}^{2}\right)\end{array}$ \\
\hline Total number of segments & 184 & 248 & 200 & 224 \\
\hline $\begin{array}{l}\text { Subjective IQ score, mean } \\
\text { 5: Very good image quality, } \\
\text { no artifacts; } \\
\text { 4: Fully diagnostic image quality, } \\
\text { no artifacts; } \\
\text { 3: diagnostic image quality, } \\
\text { minor artifacts; } \\
\text { 2: Poor image quality; severe } \\
\text { artifacts; } \\
\text { 1: Non-diagnostic segment }\end{array}$ & $4.4 \pm 0.6$ & $4.5 \pm 0.5$ & $4.6 \pm 0.6$ & $4.5 \pm 0.6$ \\
\hline Diagnostic image quality & $\begin{array}{l}100 \% \\
(184 / 184)\end{array}$ & $\begin{array}{l}100 \% \\
(248 / 248)\end{array}$ & $\begin{array}{l}100 \% \\
(200 / 200)\end{array}$ & $\begin{array}{l}100 \% \\
(224 / 224)\end{array}$ \\
\hline $\mathrm{DLP}(\mathrm{mGy})$, mean $\pm \mathrm{SD}$ & $74.3 \pm 16.5$ & $114.9 \pm 43.4$ & $123.4 \pm 35.2$ & $187.1 \pm 54.4$ \\
\hline $\begin{array}{l}\text { Effective dose }(\mathrm{mSv}) \text { median, } \\
{[25-75 \text { percentile] }}\end{array}$ & $1.1[0.9-1.2]$ & $1.7[1.0-2.0]$ & $1.6[1.4-1.9]$ & $2.7[2.1-3.0]$ \\
\hline
\end{tabular}

analysis provides for the severity and range of CAD while showing the location of the disease. This complementary information becomes significant for clinical decision-making and, if necessary, for selecting the appropriate revascularization procedure (percutaneous intervention or bypass surgery) for patients.

In the obese group of patients, the low dose protocol was also feasible on the same result in radiation dose reduction around $41 \%$ (Table 2). Image quality was found to be diagnostically acceptable in all cases. To assess and compare the accuracy of interventional and non-interventional methods, interventional coronary arteries angiography (CAA) was performed for 27 patients after CTCA: in overweight patients $80-\mathrm{kV}$ group - 8 patients; $100-\mathrm{kV}-7$ patients; in obese patients $100-\mathrm{kV}$ group -6 patients, $120-\mathrm{kV}-6$ patients. A high correlation was found for obstructive coronary artery stenosis (>50\%; kappa 0.9). In the interventional CAA group, the sensitivity and specificity of CTCA were $90 \%$ and $78 \%$, respectively, comparing all groups in the diagnosis of significant stenosis.

\subsection{Qualitative Image Quality Analysis}

A total of 856 segments were qualitatively assessed: 184 segments [80-kV] versus 248 segments [100-kV] in the overweight patients group and 200 segments [100-kV] versus 224 segments $[120-\mathrm{kV}]$ in the obese patients group. There were no statistically significant differences in subjective image quality assessment between the two groups. As seen in data represented in Table 2, all segments were deemed as diagnostic, with an overall diagnostic image quality of $100 \%$ and no artifacts observed. We performed a comparison of qualitative image quality by two independent observers and the results showed perfect agreement with ICC of 0.8 . As shown in Fig. 2, the image quality regarding noise and 

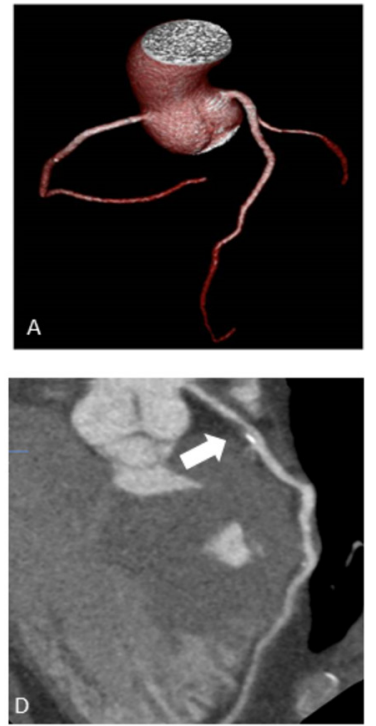
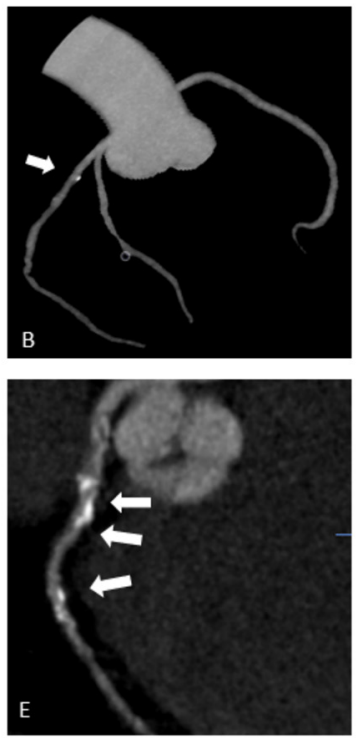
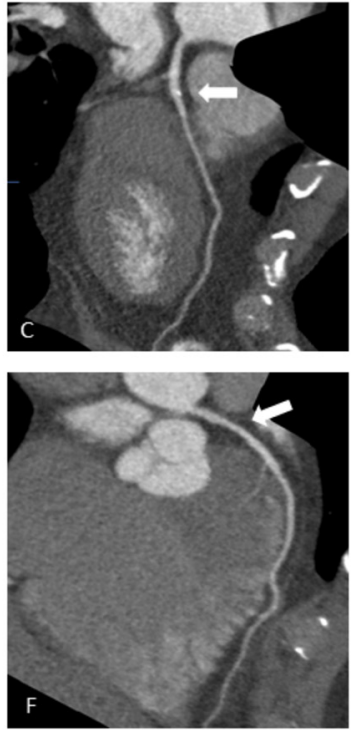

Fig. 2. Coronary arteries' scanning images (Group 2 - obese patients). A - Coronary arteries' 3D reconstruction. Patient's BMI $32.5 \mathrm{~kg} / \mathrm{m}^{2} ; 100-\mathrm{kV}$; Effective dose $1.8 \mathrm{mSv}$.

B - Coronary arteries' 3D reconstruction. Patient's BMI $31.2 \mathrm{~kg} / \mathrm{m}^{2} ; 100-\mathrm{kV}$; Effective dose $1.6 \mathrm{mSv}$; Calcified plaque in LAD S6 (labelled by white arrow).

C - Multiplanar curved reconstruction of LAD coronary artery. LAD S6 mixed plaque (labelled by white arrow), $30 \%$ stenosis. Patient's BMI $32.5 \mathrm{~kg} / \mathrm{m}^{2}, 100-\mathrm{kV}$.

D - Multiplanar curved reconstruction of LAD coronary artery. LAD S6 mixed plaque (labelled by white arrow), $75 \%$ stenosis. Patient's BMI $32.5 \mathrm{~kg} / \mathrm{m}^{2}, 100-\mathrm{kV}$.

E - Multiplanar curved reconstruction of LAD coronary artery. LAD S6, S7, S8 multiple calcified plaque (labelled by white arrow), stenosis 50-95\%. Patient's BMI $32.7 \mathrm{~kg} / \mathrm{m}^{2}, 100-\mathrm{kV}$.

F - Multiplanar reconstruction of LAD coronary artery. LAD S7 non calcified plaque (labelled by white arrow), stenosis $50 \%$. Patient's BMI $30.5 \mathrm{~kg} / \mathrm{m}^{2}, 120-\mathrm{kV}$

opacification in the obese group with ultra-low $\mathrm{kV}$ was not significantly altered, it maintained the possibility to identify the structure of plaque - calcified, non-calcified, multiple calcified, mixed, and defined stenosis grade.

Our study also demonstrates that in overweight and obese patients, low dose protocol affords radiation dose reduction of $35 \%$ and $41 \%$, respectively. In all cases, image quality was found to be diagnostically acceptable.

\subsection{Quantitative Image Quality Analysis}

Table 3 illustrates the mean contrast-to-noise and signal-to-noise ratios in groups as determined in 8 different coronary segments. The noise level in overweight patients group (group 1) scanned with a tube $80-\mathrm{kV}$ was higher compared to the patients scanned at $100-\mathrm{kV}(60.3 \pm 13.5$ versus $40.4 \pm 15.6 \mathrm{HU}$ ). In obese patients group (group 2) noise level was similar in low dose protocol, scanned with a tube 100-kV, compared to the patients scanned at $120-\mathrm{kV}(34.6 \pm 5.5$ versus $34.4 \pm 10.8 \mathrm{HU})$. The mean contrast-to-noise 
Table 3

Mean contrast-to-noise ratio (CNR) and signal-to-noise ratio (SNR) in Group 1 and Group 2 patients, $p<0.05$. RCA - right coronary artery; LAD - left anterior descending artery; LCX - left circumflex artery.

\begin{tabular}{|c|c|c|c|c|c|}
\hline & \multicolumn{2}{|c|}{ Group 1 (overweight) } & \multicolumn{2}{|c|}{ Group 2 (obese) } & \multirow[b]{2}{*}{$p$-value } \\
\hline & $80-\mathrm{kV}$ & $100-\mathrm{kV}$ & $100-\mathrm{kV}$ & $120-\mathrm{kV}$ & \\
\hline \multicolumn{6}{|c|}{ Proximal RCA } \\
\hline SNR & $11.0 \pm 3.1$ & $14.2 \pm 5.6$ & $13.5 \pm 2.7$ & $12.8 \pm 4.0$ & $p<0.05$ \\
\hline CNR & $9.8 \pm 3.1$ & $12.0 \pm 5.1$ & $11.1 \pm 2.8$ & $10.7 \pm 3.8$ & $p<0.05$ \\
\hline \multicolumn{6}{|c|}{ Distal RCA } \\
\hline SNR & $9.5 \pm 2.8$ & $11.9 \pm 5.6$ & $10.2 \pm 3.2$ & $10.4 \pm 4.4$ & $p<0.05$ \\
\hline CNR & $8.3 \pm 2.7$ & $9.7 \pm 5.3$ & $7.8 \pm 3.1$ & $8.3 \pm 4.4$ & $p<0.05$ \\
\hline \multicolumn{6}{|c|}{ Left main } \\
\hline SNR & $10.8 \pm 3.2$ & $15.7 \pm 6.1$ & $15.4 \pm 3.1$ & $14.8 \pm 4.6$ & $p<0.05$ \\
\hline CNR & $9.6 \pm 3.2$ & $13.5 \pm 5.5$ & $13.0 \pm 3.3$ & $12.6 \pm 4.5$ & $p<0.05$ \\
\hline \multicolumn{6}{|c|}{ Proximal LAD } \\
\hline SNR & $10.7 \pm 2.4$ & $15.1 \pm 6.2$ & $14.5 \pm 2.1$ & $14.1 \pm 4.7$ & $p<0.05$ \\
\hline CNR & $9.5 \pm 2.5$ & $12.9 \pm 5.7$ & $12.1 \pm 2.0$ & $11.9 \pm 4.6$ & $p<0.05$ \\
\hline \multicolumn{6}{|c|}{ Distal LAD } \\
\hline SNR & $8.3 \pm 2.9$ & $10.9 \pm 4.2$ & $12.2 \pm 3.0$ & $11.5 \pm 3.5$ & $p<0.5$ \\
\hline CNR & $7.1 \pm 2.9$ & $8.7 \pm 3.7$ & $9.8 \pm 3.0$ & $9.4 \pm 3.3$ & $p<0.05$ \\
\hline \multicolumn{6}{|c|}{ First diagonal } \\
\hline SNR & $6.7 \pm 1.8$ & $8.4 \pm 4.6$ & $9.9 \pm 2.8$ & $9.4 \pm 4.6$ & $p<0.05$ \\
\hline CNR & $5.5 \pm 1.8$ & $5.9 \pm 4.2$ & $7.5 \pm 2.5$ & $7.2 \pm 4.6$ & $p<0.05$ \\
\hline \multicolumn{6}{|c|}{ Proximal LCX } \\
\hline SNR & $9.9 \pm 2.7$ & $13.5 \pm 5.4$ & $12.8 \pm 3.5$ & $12.8 \pm 3.7$ & $p<0.05$ \\
\hline CNR & $8.8 \pm 2.7$ & $11.3 \pm 4.8$ & $10.4 \pm 3.5$ & $10.6 \pm 3.5$ & $p<0.05$ \\
\hline \multicolumn{6}{|c|}{ Distal LCX } \\
\hline SNR & $7.7 \pm 1.9$ & $9.1 \pm 3.4$ & $10.3 \pm 2.6$ & $10.7 \pm 3.4$ & $p<0.05$ \\
\hline CNR & $6.5 \pm 1.9$ & $6.9 \pm 2.9$ & $8.0 \pm 2.4$ & $8.5 \pm 3.2$ & $p<0.05$ \\
\hline
\end{tabular}

ratio (CNR) and signal-to-noise ratio (SNR) were higher at $100-\mathrm{kV}$ versus $80-\mathrm{kV}$ in overweight patients (CNR $8.14 \pm 3.0[80-\mathrm{kV}]$ versus $10.1 \pm 5.4[100-\mathrm{kV}], p<0.05$ and SNR $9.3 \pm 3.0[80-\mathrm{kV}]$ vs. $12.3 \pm 5.8[100-\mathrm{kV}], p<0.05)$. Significantly higher CNR and SNR values were observed in all coronary segments. In obese patients group CNR and SNR were similar at $120-\mathrm{kV}$ and $100-\mathrm{kV}(\mathrm{CNR} 10.0 \pm 3.4[100-\mathrm{kV}]$ vs. $9.9 \pm 4.3[120-\mathrm{kV}]$, $p<0.05$ and SNR $12.4 \pm 3.4[100-\mathrm{kV}]$ versus $12.0 \pm 4.4[100-\mathrm{kV}], p<0.05)$. In obese patients group, no significant CNR and SNR difference was found comparing low dose and standard scanning protocols.

\section{Discussion}

Various meta-analyses and studies have been carried out on the diagnostic priorities of CTCA versus CAA for the detection or exclusion of CAD diagnosis. In most cases, the results show excellent sensitivity of CTCA but slightly lower specificity. In addition, it's important to estimate no-, single- or multi-vessel disease, because a patient's survival will not decrease by revascularization therapy and optimal medical therapy will result in a similar outcome. According to Meijboom et al. (2008), the diagnostic performance 
of CTCA on a per-vessel level is not as good: sensitivity is 75-95\% and specificity is 77-90\% (Prakash et al., 2010). Other literature sources indicate a rather good sensitivity (86\%-93\%) and excellent specificity $(96 \%-97 \%)$ to detect significant coronary lesions by CTCA (Abdulla et al., 2007; Mowatt et al., 2008; Stein et al., 2008). Our data on sensitivity and specificity of CTCA founded $90 \%$ and $78 \%$, respectively, are consistent with other data.

One of the widely discussed limitations of radiological investigation methods is the increased risk of lung and breast cancer genesis (Einstein et al., 2007). Several strategies are being developed to reduce the radiation dose by tube current modulation, low-tube voltage imaging, modification of reconstruction algorithms (Hausleiter et al., 2010; Hosch et al., 2011; Prakash et al., 2010). In our study, we tested the feasibility of low dose protocols $(80-\mathrm{kV}$ and $100-\mathrm{kV})$ by decreasing tube kilovoltage, depending on BMI and demonstrated a significant reduction of radiation dose $35 \%$ and $41 \%$ versus standard $100-\mathrm{kV}$ and $120-\mathrm{kV}$ protocols: $(1.1 \mathrm{mSv}$ [0.9-1.2] $(80-\mathrm{kV})$ versus $1.7 \mathrm{mSv}$ [1.0-2.0] $(100-\mathrm{kV})$ and $1.6 \mathrm{mSv}$ [1.4-1.9] (100-kV) versus $2.7 \mathrm{mSv}$ [2.1-3.0] (120-kV)). Quantitative measures of quality showed an increase in image noise and a decrease in CNR and SNR in the 80-kV group, but the diagnostic image quality was preserved in the $80-\mathrm{kV}$ group. Similar results were obtained in the $100-\mathrm{kV}$ obese patients group. This reduction in tube potential resulted in reduced radiation exposure while maintaining image quality.

The strategy to decrease radiation exposure is to employ noise-reducing iterative reconstruction algorithms while reducing tube potential. Iterative reconstructions (IR) can produce higher resolution images and reduced imaging artifacts and have been adopted by CTCA in recent years (Leipsic et al., 2012). Using IR with CTCA leads to a significant reduction of radiation dose compared to filtered back projection (FBP) (Abdulla et $a l .$, 2007). The use of adaptive iterative dose reduction in three-dimensions (AIDR3D) reconstruction is capable of achieving up to $50 \%$ radiation reduction while maintaining comparable image quality compared to FBP techniques (Chen et al., 2013). The combination of AIDR3D and automated exposure control markedly reduced radiation exposure by utilization of low tube potential while decreasing the associated noise problem (Wong et al., 2014). It is recommended that in the future more types of IR should be included to verify findings. The different parameters of using IR should be emphasized particularly on blending options and approaches, so that achievable dose reduction can be maximized. This may help to determine the most effective approach of using IR in CCTA to reduce patient radiation dose as well as maintain the diagnostic image quality (Abdullah et al., 2016). Our study perfectly demonstrates that in evaluated overweight and obese patients, the low dose protocol resulted in radiation dose reduction of $35 \%$ and $41 \%$, while image quality was diagnostically acceptable in all cases.

The image quality of CTCA depends on multiple key aspects of the imaging process. There are CT-related parameters, including temporal resolution, tube potential, tube current, and scan reconstruction methods. Our study was performed with the first generation 320 detectors row CT scanner, with the gantry rotation time of $350 \mathrm{~ms}$ in combination with up to $16 \mathrm{~cm}$ cranial-caudal volume coverage per rotation, which enables single heartbeat acquisitions. These features give the opportunity to get a homogeneous view of the entire 
heart and avoid stepwise artifacts. A heart rate up to 65 beats per minute is optimal for diagnostic image quality for this type of scanner. On the other hand, patient-related factors such as heart rate during acquisition, patient compliance, and BMI also influence image quality. Good heart rate control with $\beta$-blockers remains an essential strategy in achieving good image quality and low radiation dose (Wong et al., 2014).

The use of a lower tube kilovoltage setting for imaging is associated with a significant increase in image signal and contrast. This increase is a benefit of imaging with a reduced tube voltage and may improve visualization of contrast-enhanced structures and may potentially permit the use of less contrast volume to obtain similar signal and contrast as imaging with a higher tube voltage. The increased signal and contrast can be attributed to the characteristics of iodinated contrast material, which provides increased signal attenuation and higher contrast to other structures in the setting of reduced tube voltage. This X-ray fundamental results from the fact that $80-\mathrm{kVp}$ imaging is closer to the K-edge (absorption capability) of iodine. In addition to the differences in signal and contrast, image noise at $80-\mathrm{kVp}$ compared with $100-\mathrm{kVp}$ was increased because of the reduced penetration of photons at lower energy. This increase in noise is a potential disadvantage of $80-\mathrm{kVp}$ imaging because the greater the noise the lower the overall image quality. It is also worth noting that the increase in the noise was proportionally larger than the increase in the signal and contrast, resulting in net decreases in the signal-to-noise and contrast-to-noise ratios (LaBounty et al., 2011).

After the iterative reconstruction algorithms have been applied, lowering the tube potential has become a well-recognized strategy to reduce the radiation dose of CTCA. Tube potential determines the energy of the X-ray beam, which affects its penetrability and noise (Meijboom et al., 2008). A subsequent multicentre trial conducted by Labounty and colleagues demonstrated that a radiation dose reduction of $47 \%$ can be achieved by reducing the tube potential from $100-\mathrm{kV}$ to $80-\mathrm{kV}$ (LaBounty et al., 2011), also similar results have been described in smaller, single-centre studies (Halliburton et al., 2011). A reduction in tube potential from $120-\mathrm{kV}$ to $100-\mathrm{kV}$ results in a $31 \%$ reduction in radiation dose, however, it also results in a $20 \%$ increase in the noise if all other parameters are unchanged (Wong et al., 2014). 100-kV protocols result in significantly lower radiation, while mean vascular attenuation is significantly higher (Takx et al., 2017).

Low tube settings can improve the contrast of the coronary artery; however, they also can increase blooming artifacts from coronary calcifications. In practice, to counter the negative effect of image noise by using lower tube potential, an increase in tube current may be necessary.

\section{Limitations}

This is a single centre experience with a limited number of patients. The true coronary stenosis could not be proved because not every patient underwent invasive CAA. The diagnostic accuracy of the new generation 320-detector row CT compared to CAA will need further evaluation but studies showed comparable results (Dewey et al., 2009; Nasis et al., 2010; Sun et al., 2012). 


\section{Conclusions}

The healthcare sector benefits significantly from the application of data science in medical imaging. Computational and image processing techniques need to move forward to bring direct benefit to clinical practice. This paper presents a study of the diagnostic role of CTCA using optimal scanning parameters and the impact of low kilovoltage CTCA on qualitative and quantitative image parameters and radiation dose in overweight and obese patients. A consolidation of knowledge in the field of image processing and medicine has been used, and the assessment of the performance in clinical conditions has been performed.

In this paper, a low radiation dose analysis of the CTCA was carried out, especially involving overweight or really obese patients. According to the results, the CTCA images obtained using the 320-CT scanner were of appropriate quality and required a lower dose of radiation in overweight patients using $80-\mathrm{kV}$ when compared with the $100-\mathrm{kV}$ standard scanning protocol and in obese patients using $100-\mathrm{kV}$ compared with the $120-\mathrm{kV}$ standard scanning protocol. The results demonstrate low $\mathrm{kV}$ scanning protocol potential in significant dose reduction in evaluated overweight and obese patients accordingly $35 \%$ and $41 \%$ with diagnostically acceptable and high image quality in all cases. The results of this study lead us to optimize and individualize CT scanning protocols and reduce radiation dose.

\section{References}

Abbara, S., Blanke, P., Maroules, C.D., Cheezum, M., Choi, A.D., Han, B.K., Marwan, M., Naoum, C., Norgaard, B.L., Rubinshtein, R., Schoenhagen, P., Villines, T., Leipsic, J. (2016). SCCT guidelines for the performance and acquisition of coronary computed tomographic angiography: a report of the society of Cardiovascular Computed Tomography Guidelines Committee: endorsed by the North American Society for Cardiovascular Imaging (NASCI). Journal of Cardiovascular Computed Tomography, 10(6), 435-449.

Abdulla, J., Abildstrom, S.Z., Gotzsche, O., Christensen, E., Kober, L., Torp-Pedersen, C. (2007). 64-multislice detector computed tomography coronary angiography as potential alternative to conventional coronary angiography: a systematic review and meta-analysis. European Heart Journal, 28(24), 3042-3050.

Abdullah, K.A., McEntee, M.F., Reed, W., Kench, P.L. (2016). Radiation dose and diagnostic image quality associated with iterative reconstruction in coronary CT angiography: a systematic review. Journal of Medical Imaging and Radiation Oncology, 60(4), 459-468.

Achenbach, S., Marwan, M., Ropers, D., Schepis, T., Pflederer, T., Anders, K., Kuettner, A., Daniel, W.G., Uder, M., Lell, M.M. (2009). Coronary computed tomography angiography with a consistent dose below $1 \mathrm{mSv}$ using prospectively electrocardiogram-triggered high-pitch spiral acquisition. European Heart Journal, 31(3), 340-346.

Achenbach, S., Goroll, T., Seltmann, M., Pflederer, T., Anders, K., Ropers, D., Daniel, W.G., Uder, M., Lell, M., Marwan, M. (2011). Detection of coronary artery stenoses by low-dose, prospectively ECG-triggered, high-pitch spiral coronary CT angiography. JACC: Cardiovascular Imaging, 4(4), 328-337.

Bilinskas, M.J., Dzemyda, G., Trakymas, M. (2017). Feature-based registration of thorax CT scan slices. Informatica, 28(3), 439-452.

Bilinskas, M.J., Dzemyda, G., Trakymas, M. (2018). Approximation of the ribs-bounded contour in a tomography scan slice. International Journal of Information Technology \& Decision Making, 17(01), 83-102.

Bittencourt, M.S., Schmidt, B., Seltmann, M., Muschiol, G., Ropers, D., Daniel, W.G., Achenbach, S. (2011). Iterative reconstruction in image space (IRIS) in cardiac computed tomography: initial experience. The International Journal of Cardiovascular Imaging, 27(7), 1081-1087. 
Budginaitè, E., Morkūnas, M., Laurinavičius, A., Treigys, P. (2021). Deep learning model for cell nuclei segmentation and lymphocyte identification in whole slide histology images. Informatica, 32(1), 23-40. https://doi.org/10.15388/20-INFOR442.

Budoff, M.J., Dowe, D., Jollis, J.G., Gitter, M., Sutherland, J., Halamert, E., Scherer, M., Bellinger, R., Martin, A., Benton, R., Delago, A., Min, J.K. (2008). Diagnostic performance of 64-multidetector row coronary computed tomographic angiography for evaluation of coronary artery stenosis in individuals without known coronary artery disease: results from the prospective multicenter ACCURACY (Assessment by Coronary Computed Tomographic Angiography of Individuals Undergoing Invasive Coronary Angiography) trial. Journal of the American College of Cardiology, 52(21), 1724-1732.

Chen, M.Y., Steigner, M.L., Leung, S.W., Kumamaru, K.K., Schultz, K., Mather, R.T., Arai, A.E., Rybicki, F.J. (2013). Simulated 50\% radiation dose reduction in coronary CT angiography using adaptive iterative dose reduction in three-dimensions (AIDR3D). The International Journal of Cardiovascular Imaging, 29(5), $1167-1175$.

Dalager, M.G., Bøttcher, M., Dalager, S., Andersen, G., Thygesen, J., Pedersen, E.M., Bøtker, H.E. (2011). Imaging atherosclerotic plaques by cardiac computed tomography in vitro: impact of contrast type and acquisition protocol. Investigative Radiology, 46(12), 790-795.

Dewey, M., Zimmermann, E., Deissenrieder, F., Laule, M., Dübel, H.-P., Schlattmann, P., Knebel, F., Rutsch, W., Hamm, B. (2009). Noninvasive coronary angiography by 320-row computed tomography with lower radiation exposure and maintained diagnostic accuracy: comparison of results with cardiac catheterization in a head-to-head pilot investigation. Circulation, 120(10), 867-875.

Dzemyda, G. (2018). Data Science and Advanced Digital Technologies. In: International Baltic Conference on Databases and Information Systems, pp. 3-7. Springer.

Einstein, A.J., Henzlova, M.J., Rajagopalan, S. (2007). Estimating risk of cancer associated with radiation exposure from 64-slice computed tomography coronary angiography. Jama, 298(3), 317-323.

Engel, L.-C, Ferencik, M., Liew, G.Y., Karolyi, M., Sidhu, M.S., Lee, A.M., Wai B., Blankstein R., Abbara S., Hoffmann U., Ghoshhajra B.B. (2012). Ultra-low dose cardiac CT angiography at $80 \mathrm{kV}$ using second generation dual-source CT: assessment of radiation dose and image quality. Journal of Medical Diagnostic Methods, 1, 104.

Feuchtner, G.M., Jodocy, D., Klauser, A., Haberfellner, B., Aglan, I., Spoeck, A., Hiehs, S., Soegner, P., Jaschke, W. (2010). Radiation dose reduction by using $100-\mathrm{kV}$ tube voltage in cardiac 64-slice computed tomography: a comparative study. European Journal of Radiology, 75(1), 51-56.

Gagarina, N.V., Irwan, R., Gordina, G., Fominykh, E., Sijens, P.E. (2011). Image quality in reduced-dose coronary CT angiography. Academic Radiology, 18(8), 984-990.

Ghekiere, O., Salgado, R., Buls, N., Leiner, T., Mancini, I., Vanhoenacker, P., Dendale, P., Nchimi, A. (2017). Image quality in coronary CT angiography: challenges and technical solutions. The British Journal of Radiology, 90(1072), 20160567.

Halliburton, S.S., Abbara, S., Chen, M.Y., Gentry, R., Mahesh, M., Raff, G.L., Shaw, L.J., Hausleiter, J. (2011). SCCT guidelines on radiation dose and dose-optimization strategies in cardiovascular CT. Journal of Cardiovascular Computed Tomography, 5(4), 198-224.

Hausleiter, J., Martinoff, S., Hadamitzky, M., Martuscelli, E., Pschierer, I., Feuchtner, G.M., Catalán-Sanz, P., Czermak, B., Meyer, T.S., Hein, F., Bischoff, B., Kuse, M., Schómig, A., Achenbach, S. (2010). Image quality and radiation exposure with a low tube voltage protocol for coronary CT angiography: results of the PROTECTION II Trial. JACC: Cardiovascular Imaging, 3(11), 1113-1123.

Heilbron, B., Leipsic, J. (2010). Submillisievert coronary computed tomography angiography using adaptive statistical iterative reconstruction-a new reality. The Canadian Journal of Cardiology, 26(1), 35.

Hosch, W., Heye, T., Schulz, F., Lehrke, S., Schlieter, M., Giannitsis, E., Kauczor, H.-U., Katus, H.A., Korosoglou, G. (2011). Image quality and radiation dose in 256-slice cardiac computed tomography: comparison of prospective versus retrospective image acquisition protocols. European Journal of Radiology, 80(1), $127-135$.

Huda, W., Scalzetti, E.M., Levin, G. (2000). Technique factors and image quality as functions of patient weight at abdominal CT. Radiology, 217(2), 430-435.

Kalender, W.A., Deak, P., Kellermeier, M., van Straten, M., Vollmar, S.V. (2009). Application-and patient sizedependent optimization of X-ray spectra for CT. Medical Physics, 36(3), 993-1007.

Kielaite-Gulla, A., Samuilis, A., Raisutis, R., Dzemyda, G., Strupas, K. (2021). The concept of AI-based algorithm: analysis of CEUS images and HSPs for identification of early parenchymal changes in severe acute pancreatitis. Informatica, 32(2), 305-319. https://doi.org/10.15388/21-INFOR453. 
LaBounty, T.M., Leipsic, J., Poulter, R., Wood, D., Johnson, M., Srichai, M.B., Cury, R.C., Heilbron, B., Hague, C., Lin, F.Y., Taylor, C., Mayo, J.R., Thakur, Y., Earls, J.P., John Mancini, G.B., Dunning, A., Gomez, M.J., Min, J.K. (2011). Coronary CT angiography of patients with a normal body mass index using $80 \mathrm{kVp}$ versus $100 \mathrm{kVp}$ : a prospective, multicenter, multivendor randomized trial. American Journal of Roentgenology, 197(5), 860-867.

Leipsic, J., Heilbron, B.G., Hague, C. (2012). Iterative reconstruction for coronary CT angiography: finding its way. The International Journal of Cardiovascular Imaging, 28(3), 613-620.

Leschka, S., Stolzmann, P., Schmid, F.T., Scheffel, H., Stinn, B., Marincek, B., Alkadhi, H., Wildermuth, S. (2008). Low kilovoltage cardiac dual-source CT: attenuation, noise, and radiation dose. European Radiology, 18(9), 1809-1817.

Meijboom, W.B. (2009). Diagnostic performance of 64-slice CT coronary angiography. PhD thesis, Erasmus MC: University Medical Center Rotterdam.

Meijboom, W.B., Meijs, M.F., Schuijf, J.D., Cramer, M.J., Mollet, N.R., van Mieghem, C.A., Nieman, K., van Werkhoven, J.M., Pundziute, G., Weustink, A.C., de Vos, A.M., Pugliese, F., Rensing, B., Wouter Jukema, J., Bax, J.J., Prokop, M., Doevendans, P.A., Hunink, M.G.M., Krestin, G.P., de Feyter, P.J. (2008). Diagnostic accuracy of 64-slice computed tomography coronary angiography: a prospective, multicenter, multivendor study. Journal of the American College of Cardiology, 52(25), 2135-2144.

Mowatt, G., Cook, J.A., Hillis, G.S., Walker, S., Fraser, C., Jia, X., Waugh, N. (2008). 64-Slice computed tomography angiography in the diagnosis and assessment of coronary artery disease: systematic review and meta-analysis. Heart, 94(11), 1386-1393.

Nasis, A., Leung, M.C., Antonis, P.R., Cameron, J.D., Lehman, S.J., Hope, S.A., Crossett, M.P., Troupis, J.M., Meredith, I.T., Seneviratne, S.K. (2010). Diagnostic accuracy of noninvasive coronary angiography with 320-detector row computed tomography. The American Journal of Cardiology, 106(10), 1429-1435.

Oda, S., Utsunomiya, D., Funama, Y., Awai, K., Katahira, K., Nakaura, T., Yanaga, Y., Namimoto, T., Yamashita, Y. (2011). A low tube voltage technique reduces the radiation dose at retrospective ECG-gated cardiac computed tomography for anatomical and functional analyses. Academic Radiology, 18(8), 991-999.

Pflederer, T., Rudofsky, L., Ropers, D., Bachmann, S., Marwan, M., Daniel, W.G., Achenbach, S. (2009). Image quality in a low radiation exposure protocol for retrospectively ECG-gated coronary CT angiography. American Journal of Roentgenology, 192(4), 1045-1050.

Pocè, I., Arsenjeva, J., Kielaitè-Gulla, A., Samuilis, A., Strupas, K., Dzemyda, G. (2021). Pancreas segmentation in CT images: state of the art in clinical practice. Baltic Journal of Modern Computing, 9(1), 25-34.

Prakash, P., Kalra, M.K., Digumarthy, S.R., Hsieh, J., Pien, H., Singh, S., Gilman, M.D., Shepard, J.-A.O. (2010). Radiation dose reduction with chest computed tomography using adaptive statistical iterative reconstruction technique: initial experience. Journal of Computer Assisted Tomography, 34(1), 40-45.

Stein, P.D., Yaekoub, A.Y., Matta, F., Sostman, H.D. (2008). 64-slice CT for diagnosis of coronary artery disease: a systematic review. The American Journal of Medicine, 121(8), 715-725.

Stocker, T.J., Deseive, S., Leipsic, J., Hadamitzky, M., Chen, M.Y., Rubinshtein, R., Heckner, M., Bax, J.J., Fang, X.-M., Grove, E.L., Lesser, J., Maurovich-Horvat, P., Otton, J., Shin, S., Pontone, G., Marques, H., Chow, B., Nomura, C.H., Tabbalat, R., Schmermund, A., Kang, J.-W., Naoum, C., Atkins, M., Martuscelli, E., Massberg, S., Hausleite, J. (2018). Reduction in radiation exposure in cardiovascular computed tomography imaging: results from the PROspective multicenter registry on radiaTion dose Estimates of cardiac CT angIOgraphy iN daily practice in 2017 (PROTECTION VI). European Heart Journal, 39(41), 3715-3723.

Sun, G., Li, M., Jiang, X., Li, L., Peng, Z., Li, G., Xu, L. (2012). 320-detector row CT coronary angiography: effects of heart rate and heart rate variability on image quality, diagnostic accuracy and radiation exposure. The British Journal of Radiology, 85(1016), 388-394.

Takx, R.A., Krissak, R., Fink, C., Bachmann, V., Henzler, T., Meyer, M., Nance, J.W., Schoenberg, S.O., Apfaltrer, P. (2017). Low-tube-voltage selection for triple-rule-out CTA: relation to patient size. European Radiology, 27(6), 2292-2297.

Wang, D., Hu, X.-H., Zhang, S.-Z., Wu, R.-Z., Xie, S.-S., Chen, B., Zhang, Q.-W. (2012). Image quality and dose performance of $80 \mathrm{kV}$ low dose scan protocol in high-pitch spiral coronary CT angiography: feasibility study. The International Journal of Cardiovascular Imaging, 28(2), 415-423.

Wong, D.T., Soh, S.Y., Ko, B.S., Cameron, J.D., Crossett, M., Nasis, A., Troupis, J., Meredith, I.T., Seneviratne, S.K. (2014). Superior CT coronary angiography image quality at lower radiation exposure with second generation 320-detector row CT in patients with elevated heart rate: a comparison with first generation 320-detector row CT. Cardiovascular Diagnosis and Therapy, 4(4), 299. 
D. Golubickas is a physician radiologist, $\mathrm{MD}, \mathrm{PhD}$, member of Kaunas Region Society of Radiologists, Lithuanian Radiologists' Association. Member of European Association of Radiologists (ESR). Field of interest - evaluating the possibilities of different radiological research methods in detection of initial and early pathological changes.

S. Lukosevicius is a physician radiologist, $\mathrm{MD}, \mathrm{PhD}$, member of Kaunas Region Society of Radiologists, Lithuanian Radiologists' Association. Member of European Association of Radiologists (ESR). Scientific work is performed in Lithuanian University of Health Sciences, evaluating the possibilities of different radiological research methods in detection of vascular, neurological, oncological diseases, identifying both initial and early pathological changes of various kinds.

V. Tamakauskas is a head of Interventional Angiology department at Siauliai Hospital, Heart and Vascular Centre. Board member of Lithuania National Interventional Cardiology Association. Member of Lithuania Society of Cardiology, member of European Society of Cardiology (ESC) and European Association of Percutaneous Cardiovascular Interventions (EAPCI). Scientific work is focused on analysis of effectiveness, adverse effects and genetical factors of antiplatelet drugs in patients after acute coronary syndrome, especially on obese and diabetic patients.

L. Dobrovolskiene is a physician radiologist, MD, PhD. Member of Kaunas Region Society of Radiologists (KRSR) and Lithuanian Radiologists Association. Member of the European Association of Radiologists (ESR), member of European Society of Thoracic Imaging (ESTI). Scientific work is performed in the field of thoracic and cardiovascular imaging evaluating the possibilities of computed tomography and magnetic resonance imaging in early detection of different cardiovascular and thoracal pathological changes, analysis the influence of contrast media using on the quality of radiological images.

I. Baseviciene, Lithuanian University of Health Sciences (LUHS). Member of Kaunas Region Society of Radiologists (KRSR), Lithuanian Radiologists Association and European Association of Radiologists (ESR). Field of interest - evaluation of different radiological research methods used on gynaecology patients, especially for obese patients, identifying both initial and early pathological changes of various kinds.

L. Grib is a head of the University Clinic of Cardiology within Public Health Hospital "Sf. Treime" and professor in State University of Medicine and Pharmacy "Nicolae Testemitanu". Member of the Society of Cardiologists of the Republic of Moldova and Romania. Member of the European Society of Cardiology (ESC). The scientific activity is focused on obese patients and current problems of radiological etiopathogenesis evaluation, diagnosis and treatment of cardiovascular disease with various comorbidities.

N. Ragaisyte, MD, PhD, cardiologist. Member of Kaunas Region Society of Cardiologists, Lithuanian Heart Association, member of European Association of Cardiologists. Field of interest - overweight patients with coronary artery diseases and possibilities for early pathological change diagnostics by using CT scanning.

R. Leonavicius is an assoc. prof. and doctor psychiatrist at Hospital of Lithuanian University of Health Sciences Kaunas Clinics. Author and co-author of more than 16 scientific 
articles, one monograph in psychiatry and 3 educational books in psychiatry and general practice. Investigator and principal investigator of 10 clinical trials. Field of interest obese patient clinical status evaluation.

V. Medvedev is a senior researcher at the Institute of Data Science and Digital Technologies, Vilnius University. He received the doctoral degree in computer science $(\mathrm{PhD})$ from Institute of Mathematics and Informatics jointly with Vilnius Gediminas Technical University, in 2008. His research interests include artificial intelligence, neural networks, visualization of multidimensional data, dimensionality reduction, image processing, data mining and parallel computing.

V. Veikutis, MD, $\mathrm{PhD}$, cardiologist. Member of the European Association of Cardiology (ESC), Fields of interest - experimental and clinical investigations in field of destructive energies (RF, US, Laser), evaluation of the possibilities of computed tomography and magnetic resonance imaging in early detection of different cardiovascular pathologies. 